\title{
Association of cardiovascular disease with 30-day hospital readmission in Chinese patients receiving maintenance dialysis
}

\author{
Rui Chen ${ }^{1}$, Chao Yang ${ }^{1}$, Ming Zhu ${ }^{2}$, Hong Chu ${ }^{1}$, Jinwei Wang ${ }^{1}$, Bixia Gao ${ }^{1}$, Lili Liu ${ }^{1}$, Yifang Jiang ${ }^{1}$, \\ Yu Lin ${ }^{3}$, Jingyi $\mathrm{Wu}^{3}$, Guilan Kong,5, Fang Wang ${ }^{1}$, Luxia Zhang ${ }^{1,4,5}$, Minghui Zhao ${ }^{1,6}$; on behalf of the \\ China Kidney Disease Network Work Group
}

${ }^{1}$ Department of Medicine, Peking University First Hospital, Beijing, China; ${ }^{2}$ Zhongshan School of Medicine, Sun Yat-sen University, Guangzhou, China; ${ }^{3}$ Department of Epidemiology and Biostatistics, School of Public Health, Peking University, Beijing, China; ${ }^{4}$ National Institute of Health Data Science at Peking University, Beijing, China; ${ }^{5}$ Center for Data Science in Health and Medicine, Peking University, Beijing, China; ${ }^{6}$ PekingTsinghua Center for Life Science, Beijing, China

Contributions: (I) Conception and design: R Chen, C Yang, L Zhang; (II) Administrative support: None; (III) Provision of study materials or patients: C Yang, J Wang, G Kong, F Wang, L Zhang; (IV) Collection and assembly of data: C Yang, J Wang; (V) Data analysis and interpretation: R Chen, C Yang, J Wang, G Kong, F Wang, L Zhang; (VI) Manuscript writing: All authors; (VII) Final approval of manuscript: All authors.

Correspondence to: Luxia Zhang. National Institute of Health Data Science at Peking University, 38 Xueyuan Street, Haidian District, Beijing 100191, China. Email: zhanglx@bjmu.edu.cn.

Background: Previous studies have shown cardiovascular disease (CVD) to be a risk factor in the prediction of 30-day hospital readmission among patients receiving dialysis. However, studies of Asian populations are limited. In the present study, we examined the association between CVD and 30-day hospital readmission in Chinese patients receiving maintenance dialysis.

Methods: Patients receiving maintenance dialysis were identified by searching a national claims database, the China Health Insurance Research Association (CHIRA) database, using the International Classification of Diseases revision 10 (ICD-10) and items of medical service claims. Patients aged $\geq 18$ years who were discharged after index hospitalization between January 2015 and December 2015 were included in our retrospective analysis. CVD-related diagnoses were divided into three categories: coronary heart disease (CHD), heart failure (HF), and stroke. Thirty-day hospital readmission was defined as any hospital readmission within the 30 days following discharge. Logistic regression models adjusted for logit of propensity scores (PS) were used to assess the association of CVD with 30-day hospital readmission.

Results: Of 4,700 patients receiving dialysis, the 30-day hospital readmission rate was $10.4 \%$. Compared with patients without CVD, there was an increased risk of 30-day hospital readmission among maintenance dialysis patients with total CVD [odds ratio (OR): 1.33, 95\% confidence interval (CI): 1.06-1.66]. Patients with HF (OR: 1.77, CI: 1.27-2.47) and stroke (OR: 2.14, 95\% CI: 1.53-2.98) had a greater risk of 30-day hospital readmission. The fully adjusted OR of CHD for the risk of 30-day hospital readmission was 1.22 (95\% CI: 0.97-1.55).

Conclusions: CVDs, especially stroke and HF, are independent predictors of 30-day hospital readmission in Chinese patients receiving dialysis, and could help to guide interventions to improve the quality of care for these patients.

Keywords: Cardiovascular disease (CVD); dialysis; 30-day hospital readmission; China

Submitted Mar 10, 2020. Accepted for publication Dec 21, 2020.

doi: 10.21037/atm-20-2367

View this article at: http://dx.doi.org/10.21037/atm-20-2367 


\section{Introduction}

High hospital readmission rates are increasingly viewed as an unnecessary drain on social and financial resources (1). The hospital readmission rate at 30 days is an important measure of care quality, and it can also be used to predict subsequent morbidity and mortality among patients with end-stage kidney disease (ESKD) (1-6). Reducing 30-day hospital readmission rates is becoming a major focus for health systems (7). The Healthcare Cost and Utilization Project Statistical Brief indicates that the 30 -day readmission rate for general inpatients is $13.9 \%$ (8). Compared with other patients, those with ESKD have a higher rate of hospital readmission within 30 days $(5,9,10)$. Data from the United States Renal Data System (USRDS) showed that the 30-day readmission rate of ESKD patients and patients receiving hemodialysis (HD) was $34.6 \%$ and $36.6 \%$, respectively (5). In 2015, 23.2\% of dialysis patients in China were readmitted within 30 days (10). Data from the urban basic health insurance (UBHI) scheme showed that $\mathrm{HD}$ and PD patients accounted for only $0.16 \%$ and $0.02 \%$ of the population covered by UBHI, respectively; however, their medical costs accounted for $2.08 \%$ and $0.34 \%$ of the total UBMI expenditures, respectively $(5,10)$. The 30-day readmission rate in China, although lower than that in the USA, is still high, placing a considerable burden on patients and society $(5,9)$.

Cardiovascular disease (CVD), a leading cause of hospitalization and death, is common among patients receiving maintenance dialysis $(5,10)$. Accumulating evidence on dialysis patients suggests that CVD increases the risk of hospital readmission (11-14). Previous studies on HD patients have suggested that heart failure (HF) increases the risk of hospital readmission (11). Studies have shown that ischemic heart disease (IHD) and HF are associated with higher rates of hospital readmission among patients receiving peritoneal dialysis (PD) $(12,13)$. Another study found that hospital readmission rates were higher among HD and PD patients with CVD (14). However, studies on the risk of 30-day hospital readmission in Asian populations are limited. Therefore, in the present study, we examined the association between CVD and 30-day hospital readmission in Chinese patients receiving maintenance dialysis. We present the following article in accordance with the STROBE reporting checklist (available at http://dx.doi. org/10.21037/atm-20-2367).

\section{Methods}

\section{Study population}

Hospitalization records of patients receiving maintenance dialysis who were discharged from hospital during 2015 were identified within the China Health Insurance Research Association (CHIRA) database using International Classification of Disease revision 10 (ICD-10) codes (i.e., N18.000, N18.001, and N18.900) and items of medical service claim, including hemodialyzer and PD fluid (15). The CHIRA database is a repository of claims from individuals covered by UBHI. It uses a two-stage sampling design to create a national sample of claims. It contains inpatient and outpatient whole-year medical records, and includes data on patient demographics, medical events, operation procedures, prescription drugs, and detailed medical expenditure. The CHIRA database has been described in detail previously (10).

All eligible patients included in the study were aged $>18$ years and were discharged after index hospitalization between January $1^{\text {st }}, 2015$ and December $31^{\text {st }}, 2015$. Patients with maintenance $\mathrm{HD}$ and maintenance PD were defined as having maintenance dialysis. All patients who did not receive dialysis were excluded. We also excluded patients with acute kidney failure and those with a history of kidney transplantation. Patients hospitalized without a recorded admission or discharge date, those with a length of stay $>1$ but $<3$ days, or $>180$ days were also excluded (14). The flow chart of patient selection is shown in Figure 1. The study was conducted in accordance with the Declaration of Helsinki (as revised in 2013). The study was approved by the Ethics Committee of Peking University First Hospital (approval number: 2018-25), and informed consent from patients was not required due to the secondary use of deidentified patient data and identifiable personal information not being used. The procedure was performed in accordance with all relevant guidelines and regulations.

\section{Identification of CVD}

CVD was defined using information from claims records (Tables S1-S3). This information included diagnosis, ICD10 codes, the International Classification of Diseases, $9^{\text {th }}$ revision, Clinical Modification (ICD-9-CM) procedure codes, and prescription drugs. CVD was categorized into three types: coronary heart disease (CHD), HF, and stroke. 


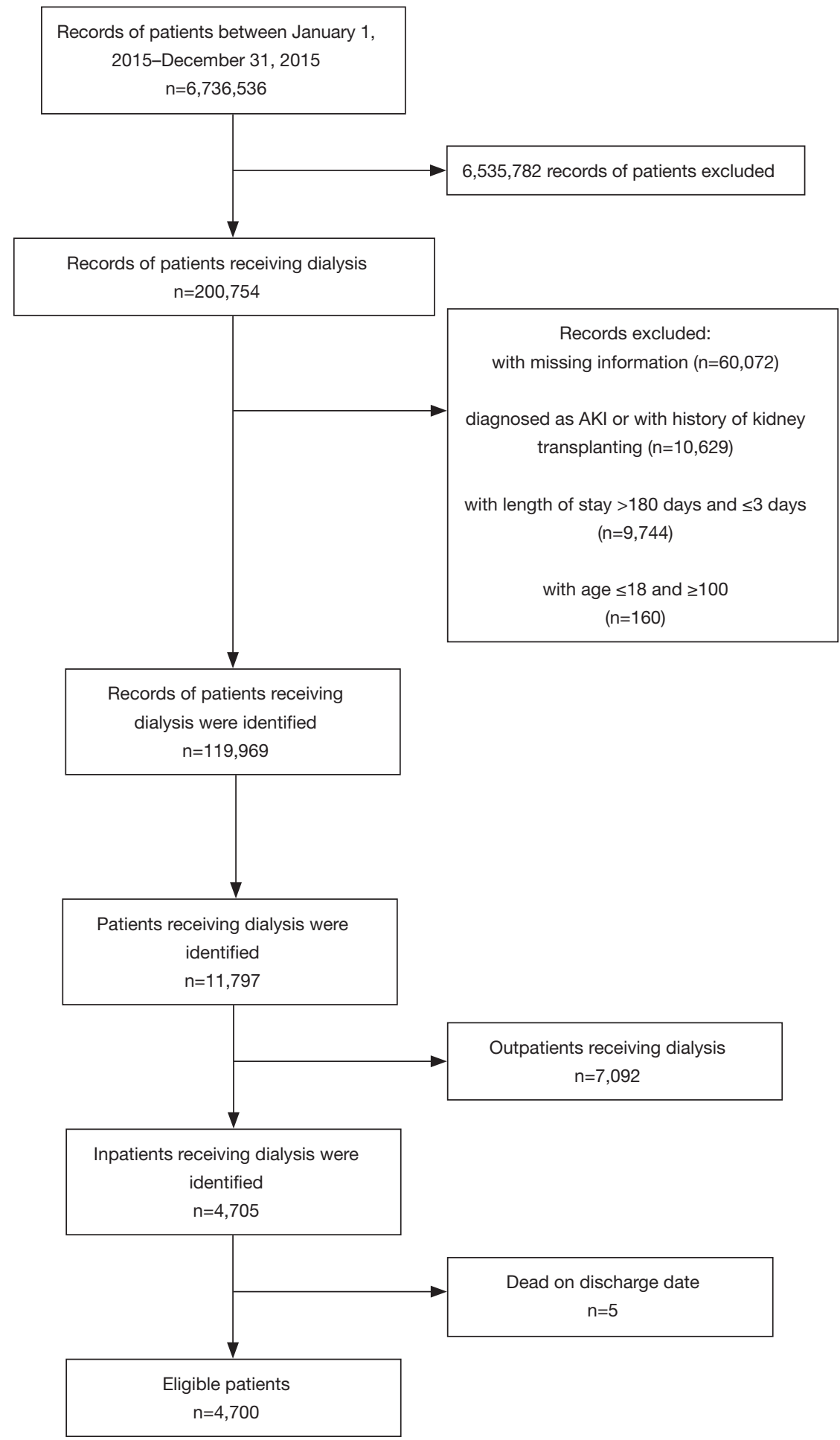

Figure 1 Selection of patients. Records represent detailed information of the medical process. Outpatients represent patients with outpatient records only. Inpatients represent patients with more than one hospitalization record. 


\section{Other covariates}

Covariates included demographic characteristics (sex and age), geographic region, type of health insurance, length of stay, hospital grade, dialysis modality, weekend discharge, season of hospital admission, comorbidities (diabetes mellitus, hypertension, infection, malignant tumor, immunosuppression, airway disease, and dyslipidemia), the need for critical care, the use of proton-pump inhibitors (PPIs), the use of anticoagulant drugs, and the use of Chinese traditional medicine. Causes of index hospitalizations were identified and categorized as CVD, infectious disease, and vascular access events.

\section{Definition of index hospital admission and 30-day hospital readmission}

Hospital admission was defined as the index admission of patients with only 1 hospitalization. If a patient had $\geq 2$ hospital admissions, the index admission used was a single admission randomly selected from the set of available admissions $(15,16)$. This measure was used to reduce the effect on the study of dependent observations. Thirty-day hospital readmission was defined as any readmission within the 30 days following discharge.

\section{Statistical analysis}

We employed covariate adjustment using propensity scores (PS). PS are the estimated probability of having CVD based on all relevant confounding variables using a logistic regression model (17). To balance the effect of comorbidities and reduce the degrees of freedom, we reduced each of the comorbidities associated with CVD to a PS to adjust for the effect of related covariates (18). For each patient, we calculated the PS for comorbidities with a logistic regression model using the variables listed in Table 1. Variables were selected using previous studies of plausible associations between comorbidities and outcome. PS were used to adjust for potential confounders in the multivariable models that assessed the association between CVDs and 30-day hospital readmission.

Characteristics of dialysis patients were exemplified using descriptive statistics. Continuous variables were expressed as means ( \pm standard deviations) or medians $\left(25^{\text {th }}\right.$ and $75^{\text {th }}$ percentiles), and categorical variables as proportions (\%). To compare the distribution of groups, the $t$-test, analysis of variance, or the Wilcoxon rank-sum test was used for continuous variables, and the $\chi^{2}$-test was used for categorical variables.

Logistic regression models were used to assess the associations between types of CVDs and 30-day hospital readmission, and the adjusted odds ratios (ORs) and 95\% confidence intervals (CIs) were calculated. Covariates included in the model included age (years, continuous; years: $18-44,45-64,65-74, \geq 75$, categorical), sex (male or female), geographic distribution (northern China or southern China), hospital grade (primary, secondary, or tertiary hospital), types of health insurance (employee insurance or resident insurance), season (spring, summer, autumn, or winter), weekend hospital discharge (yes or no), length of stay (continuous), dialysis modality (HD or PD), and PS (continuous). Statistical significance in all tests was defined as a two-tailed $\mathrm{P}$ value of $<0.05$. All analyses were undertaken using SAS version 9.4 (SAS Institute, Cary, NC, USA) and $\mathrm{R}$ version 3.6.0.

\section{Results}

\section{Patient characteristics}

From an initial cohort of 11,797 dialysis patients, 4,700 $(39.8 \%)$ eligible patients receiving maintenance dialysis were identified. Characteristics of the dialysis patients are given in Table 1. The 30-day hospital readmission rates for the 4,700 dialysis patients were $10.4 \%$ at the patient level and $23 \%$ at the admission level. The patients had a mean age of $56.4 \pm 15.6$ years, and $44.8 \%$ were female. A total of 3,980 (84.7\%) patients were receiving maintenance HD. The number of patients receiving maintenance HD with CVD was 955 (20.3\%). Compared with patients without CVD, those with CHD, HF, or stroke were more likely to be aged over 45 years old $(\mathrm{P}<0.001)$, to be from northern China $(\mathrm{P}<0.001)$, and to have multiple comorbidities, including hypertension $(\mathrm{P}<0.001$ vs. $\mathrm{P}=0.107$ vs. $\mathrm{P}<0.001$, respectively), diabetes $(\mathrm{P}<0.001$ vs. $\mathrm{P}=0.016$ vs. $\mathrm{P}<0.001$, respectively), airway disease $(\mathrm{P}<0.001$ vs. $\mathrm{P}=0.025$ vs. $\mathrm{P}<0.001$, respectively), and dyslipidemia $(\mathrm{P}<0.001$ vs. $\mathrm{P}=0.001$ vs. $\mathrm{P}<0.001$, respectively). Compared to patients without $\mathrm{CVD}$, those with $\mathrm{CHD}, \mathrm{HF}$, or stroke were more likely to receive PPI therapy $(\mathrm{P}<0.001$ vs. $\mathrm{P}=0.041$ vs. $\mathrm{P}<0.001$, respectively). Patients with CVD were also more likely to be rehospitalized for CVD than for other reasons.

\section{Comparison among patients with different types of CVDs}

The rates of hospital readmission within 30 days are shown in Table 2. Readmission within 30 days occurred more 
Table 1 Characteristics of dialysis patients by type of CVD

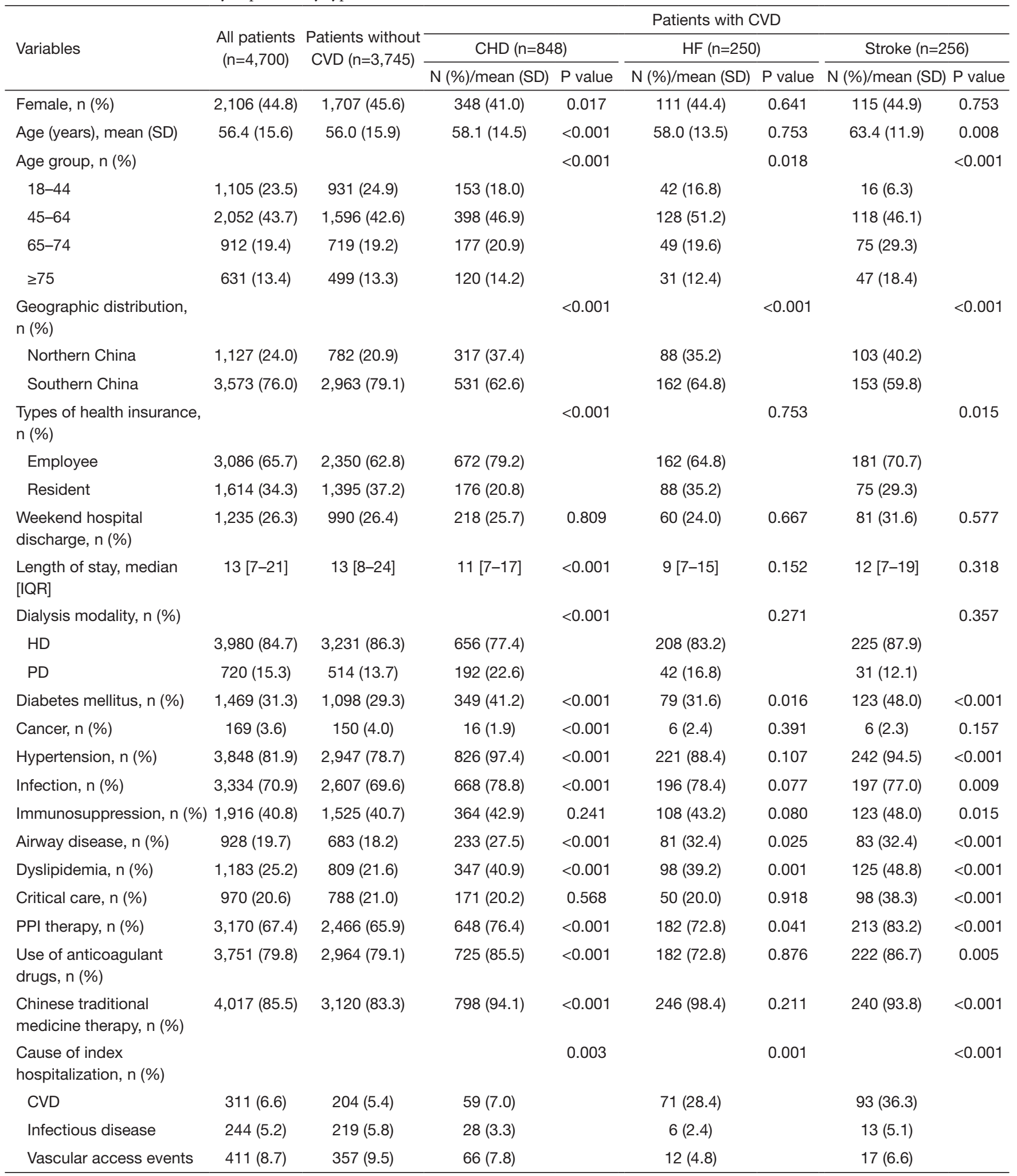

Data are presented as $\mathrm{n}(\%)$ or mean (SD) or median [IQR]. CVD, cardiovascular disease; CHD, coronary heart disease; HF, heart failure; $\mathrm{SD}$, standard deviation; IQR, interquartile range; HD, hemodialysis; PD, peritoneal dialysis; PPI, proton-pump inhibitor. 
Table 2 Thirty-day readmission rates among dialysis patients by type of CVD

\begin{tabular}{|c|c|c|c|c|c|c|c|}
\hline \multirow{3}{*}{ Characteristic } & \multirow{3}{*}{$\begin{array}{l}\text { Patients without } \\
\text { CVD }\end{array}$} & \multicolumn{6}{|c|}{ Patients with CVD } \\
\hline & & \multicolumn{2}{|c|}{$\mathrm{CHD}$} & \multicolumn{2}{|c|}{$\mathrm{HF}$} & \multicolumn{2}{|c|}{ Stroke } \\
\hline & & $\mathrm{N}(\%)$ & $P$ value & $\mathrm{N}(\%)$ & $P$ value & $\mathrm{N}(\%)$ & $P$ value \\
\hline All, n (\%) & $337(9.0)$ & $123(14.5)$ & 0.032 & $50(20.0)$ & $<0.001$ & $59(23.0)$ & $<0.001$ \\
\hline Sex, n (\%) & & & 0.145 & & 0.233 & & 0.373 \\
\hline Female & $145(8.5)$ & $46(13.2)$ & & $20(18.0)$ & & $25(21.7)$ & \\
\hline Age group, n (\%) & & & 0.017 & & 0.049 & & 0.031 \\
\hline $18-44$ & $70(7.5)$ & $14(9.2)$ & & $4(9.5)$ & & $1(6.3)$ & \\
\hline $45-64$ & $156(9.8)$ & $64(16.1)$ & & $28(21.9)$ & & $31(26.3)$ & \\
\hline Geographic distribution, n (\%) & & & $<0.001$ & & $<0.001$ & & $<0.001$ \\
\hline Northern China & $68(8.7)$ & $46(14.6)$ & & $15(17.0)$ & & $17(16.5)$ & \\
\hline Southern China & $269(9.1)$ & $77(14.5)$ & & $35(21.6)$ & & $42(27.5)$ & \\
\hline Dialysis modality, n (\%) & & & 0.063 & & 0.047 & & 0.043 \\
\hline$H D$ & $302(9.35)$ & $101(15.4)$ & & $44(21.2)$ & & $55(24.4)$ & \\
\hline PD & $35(6.81)$ & $22(11.5)$ & & $6(14.3)$ & & $4(12.9)$ & \\
\hline Diabetes mellitus, $\mathrm{n}(\%)$ & $148(13.5)$ & $45(12.9)$ & $<0.001$ & $12(15.2)$ & $<0.001$ & $35(28.4)$ & $<0.001$ \\
\hline Cancer, n (\%) & $53(35.33)$ & $1(6.3)$ & $<0.001$ & $3(50.0)$ & $<0.001$ & $2(33.3)$ & $<0.001$ \\
\hline
\end{tabular}

Data are presented as n (\%). CVD, cardiovascular disease; CHD, coronary heart disease; HF, heart failure; HD, hemodialysis; PD, peritoneal dialysis.

often in dialysis patients with CVD than patients without CVD. Patients with two comorbidities (CVD and one other comorbidity, except diabetes) were significantly more likely to be readmitted than those without CVD.

\section{CVD and the risk of 30-day hospital readmission}

Table 3 shows the logistic regression analysis of three groups for 30-day hospital readmission. Compared to patients without CVD, maintenance dialysis patients with total CVD were more likely to be readmitted within 30 days (OR: 1.33, 95\% CI: 1.06-1.66). Patients with HF (OR: 1.77, CI: $1.27-2.47$ ) or stroke (OR: $2.14,95 \%$ CI: $1.53-2.98$ ) had a higher rate of 30-day hospital readmission. The fully adjusted OR of CHD for 30-day hospital readmission was 1.22 (95\% CI: 0.97-1.55).

\section{Discussion}

To the best of our knowledge, the present study is the first to investigate the association of 30-day hospital readmission with CVD among Chinese patients receiving maintenance dialysis. Using a nationwide claims database for urban citizens in China, we found the rate of 30-day hospital readmission to be higher for patients with $\mathrm{HF}$ and stroke than for patients without CVD. Moreover, HF and stroke 
Table 3 Association between CVD and risk of 30-day readmission in patients receiving maintenance dialysis [OR (95\% CI)]

\begin{tabular}{lccccc}
\hline \multirow{2}{*}{ Models } & \multirow{2}{*}{$\begin{array}{c}\text { Patients without CVD } \\
(\mathrm{n}=2,221)\end{array}$} & All CVD $(\mathrm{n}=950)$ & \multicolumn{3}{c}{ Patients with CVD } \\
\cline { 5 - 6 } & Reference & $1.78(1.44-2.20)$ & $1.66(1.33-2.07)$ & $2.34(1.69-3.24)$ & HF $(\mathrm{n}=250)$ \\
\hline Model 1 $^{\mathrm{a}}$ & Reference & $1.75(1.42-2.16)$ & $1.61(1.31-2.03)$ & $2.31(1.67-3.20)$ & $2.69(1.94-3.72)$ \\
Model 2 $^{\mathrm{b}}$ & Reference & $1.33(1.06-1.66)$ & $1.22(0.97-1.55)$ & $1.77(1.27-2.47)$ & $2.14(1.53-2.98)$ \\
\hline
\end{tabular}

Data are presented as ORs (95\% Cls). The reference group is patients without CVD. ${ }^{a}$, model 1 was adjusted for age and sex; ${ }^{b}$, model 2 was further adjusted for age, sex, geographic distribution, hospital grade, types of health insurance, season of admission, weekend hospital discharge, length of stay, dialysis modality, and the probability of the PS using the following covariates: diabetes mellitus, cancer, hypertension, infection, immunosuppression, airway disease, dyslipidemia, critical care, use of PPI therapy, use of anticoagulant drugs, and use of Chinese traditional medicine. CVD, cardiovascular disease; OR, odds ratio; Cl, confidence interval; CHD, coronary heart disease; HF, heart failure; PS, propensity score; PPI, proton-pump inhibitor.

could independently predict 30-day hospital readmission. These findings suggest that appropriate treatment of CVD could reduce hospital readmission among dialysis patients.

Previous studies have indicated that CVD is associated with an increased risk of 30-day hospital readmission. Using data from the Nationwide Readmission Database (NRD), Chan et al. found that HF leads to an increased risk of 30-day hospital readmission in patients receiving HD (11). However, the NRD only contains data from community hospitals, and information on patients whose initial admission occurred in other states is not captured by this database; therefore, the rate of hospital readmission in HD patients may be underestimated. Data from the USRDS suggests that IHD, cerebrovascular disease, and $\mathrm{HF}$ are associated with hospital readmission in $\mathrm{PD}$ patients (12). A recent study indicated that CVDs, including arteriosclerotic heart disease, congestive $\mathrm{HF}$, and transient ischemic attack, could predict 30-day hospital readmission among patients receiving dialysis (14). These studies tended to focus on readmissions among patients receiving a single type (HD or PD) of continuous renal replacement therapy $(12,14)$. In their study, Wetmore et al. investigated hospital readmission, focusing on patients frequently hospitalized for CVD (14).

However, despite many studies on the association of CVD with 30-day readmission, there are no studies on Chinese populations receiving maintenance dialysis. It is thought that hospital readmission in Asia is significantly different to the rest of the world (5). Therefore, we evaluated the association between CVD and 30-day hospital readmission among HD and PD patients in China. Consistent with previous studies, our study indicated an increased risk of 30-day hospital readmission for HF (1.77 times) and stroke patients (2.14 times) when compared with patients without CVD.

Several potential mechanisms may underlie the correlation observed between CVD and increased risk of 30-day hospital readmission. First, CVD is associated with an increased likelihood of other chronic diseases, such as diabetes, pulmonary circulatory disease, and chronic obstructive pulmonary disease (19), and each disease serves as a potential cause of hospitalization (20). Second, sterile inflammation that occurs after stroke can result in atherosclerosis (21-24), which, as the main factor underlying most CVDs, can result in a new CVD (25). Third, with the progression of HF, anemia can arise due to iron deficiency (26), the use of angiotensin-converting enzyme (ACE) inhibitors (27), and increased hepcidin levels (28). Low hemoglobin levels serve as an independent factor of increased risk of hospital admission among dialysis patients (29). The potential biological mechanism underlying this phenomenon requires further study.

The main strength of the present study is that it evaluated 30-day hospital readmission risk among dialysis patients with control for various potential confounding variables. However, our study also has limitations. First, similar to other studies using administrative databases, claims data may contain potential errors due to the misclassification of patients. Despite this, claims data, being real-world data, are widely-used due to their authenticity. In addition to diagnosis, we used operation procedures, prescription drugs, and detailed medical expenditure to confirm patients' conditions. Second, most patients were HD patients. Third, we did not include laboratory results, dialysis age, vascular access procedures in HD patients, outpatient dialysis unit, PD subtype, severity of 
comorbidity, or other unmeasured confounders. Fourth, we did not include information about out-of-hospital deaths, which is a competing risk for hospital readmission. Fifth, we did not validate our conclusion in patients with different CHD types, and differences in the type of CHD may result in variation in prognostic outcomes. Finally, the patients in our study were beneficiaries covered by UBHI. Despite the wide coverage of UBHI in China, this raises the question of how representative our study population is.

\section{Conclusions}

CVDs, especially stroke and HF, are independent predictors of 30-day hospital readmission in Chinese patients receiving dialysis. Our findings indicate an association between CVD and 30-day hospital readmission, which may prove to be important in guiding interventions to improve quality of care. By improving medical care for CVD, the healthcare system can potentially improve outcomes and significantly reduce healthcare costs. Further intervention studies seeking to improve CVD care quality are required.

\section{Acknowledgments}

Funding: This work was supported by grants from the National Natural Science Foundation of China (Nos. 81771938, 91846101, 81301296), Peking University (Nos. BMU2018MX020, PKU2017LCX05), the National Key Technology R\&D Program of the Ministry of Science and Technology of the People's Republic of China (No. 2016YFC1305400), the University of Michigan Health System-Peking University Health Science Center Joint Institute for Translational and Clinical Research (Nos. BMU20160466, BMU2018JI012, BMU2019JI005), the Interdisciplinary Clinical Research Project of Peking University First Hospital (No. 2018CR29), and the Scientific Research Seed Fund of Peking University First Hospital (No. 2018SF069).

\section{Footnote}

Reporting Checklist: The authors have completed the STROBE reporting checklist. Available at http://dx.doi. org/10.21037/atm-20-2367

Conflicts of Interest: All authors have completed the ICMJE uniform disclosure form (available at http://dx.doi. org/10.21037/atm-20-2367). RC, CY, HC, JW, BG, LL,
YJ, YL, JW, GK, FW, LZ, and M Zhao report grants from the National Natural Science Foundation of China, Peking University, the Ministry of Science and Technology of the People's Republic of China, the University of Michigan Health System-Peking University Health Science Center Joint Institute for Translational and Clinical Research, and Peking University First Hospital. The other author has no conflicts of interest to declare.

Ethical Statement: The authors are accountable for all aspects of the work in ensuring that questions related to the accuracy or integrity of any part of the work are appropriately investigated and resolved. The study was conducted in accordance with the Declaration of Helsinki (as revised in 2013). The study was approved by The Ethics Committee of Peking University First Hospital (No. 201825). Informed consent from patients was not required due to the secondary use of deidentified patient data and identifiable personal information not being used. The procedure was performed in accordance with all relevant guidelines and regulations.

Open Access Statement: This is an Open Access article distributed in accordance with the Creative Commons Attribution-NonCommercial-NoDerivs 4.0 International License (CC BY-NC-ND 4.0), which permits the noncommercial replication and distribution of the article with the strict proviso that no changes or edits are made and the original work is properly cited (including links to both the formal publication through the relevant DOI and the license). See: https://creativecommons.org/licenses/by-nc-nd/4.0/.

\section{References}

1. Jencks SF, Williams MV, Coleman EA. Rehospitalizations among patients in the Medicare fee-for-service program. N Engl J Med 2009;360:1418-28.

2. The State Council of the People's Republic of China. The 13th Five-Year Plan for Economic and Social Development of the People's Republic of China. 2016. Available online: http://www.gov.cn/zhengce/content/2017-01/10/ content_5158488.htm

3. Zuckerman RB, Sheingold SH, Orav EJ, Ruhter J, Epstein AM. Readmissions, Observation, and the Hospital Readmissions Reduction Program. N Engl J Med 2016;374:1543-51.

4. Centers for Medicare \& Medicaid Services. Readmissions Reduction Program (HRPP). 2012. Available online: 
https://www.cms.gov/Medicare/Medicare-Fee-forService-Payment/AcuteInpatientPPS/ReadmissionsReduction-Program.html

5. Saran R, Robinson B, Abbott KC, et al. US Renal Data System 2018 Annual Data Report: Epidemiology of Kidney Disease in the United States. Am J Kidney Dis 2019;73:A7-8.

6. Plantinga LC, King L, Patzer RE, et al. Early hospital readmission among hemodialysis patients in the United States is associated with subsequent mortality. Kidney Int 2017;92:934-41.

7. Burgess JF, Hockenberry JM. Can all cause readmission policy improve quality or lower expenditures? A historical perspective on current initiatives. Health Econ Policy Law 2014;9:193-213.

8. Bailey MK, Weiss AJ, Barrett ML, et al. Characteristics of 30-day all-cause hospital readmissions, 2010-2016. Statistical Brief \#248. Rockville: Healthcare Cost and Utilization Project (HCUP) Statistical Briefs. 2019.

9. Barnett ML, Hsu J, McWilliams JM. Patient characteristics and differences in hospital readmission rates. JAMA Intern Med 2015;175:1803-12.

10. Zhang L, Zhao MH, Zuo L, et al. China Kidney Disease Network (CK-NET) 2015 Annual Data Report. Kidney Int Suppl (2011) 2019;9:e1-81.

11. Chan L, Chauhan K, Poojary P, et al. National estimates of 30-day unplanned readmissions of patients on maintenance hemodialysis. Clin J Am Soc Nephrol 2017;12:1652-62.

12. Sahlie A, Jaar B, Galarza Paez L, et al. Burden and correlates of hospital readmissions among U.S. peritoneal dialysis patients. Perit Dial Int 2019;39:261-7.

13. Chan L, Poojary P, Saha A, et al. Reasons for admission and predictors of national 30-day readmission rates in patients with end-stage renal disease on peritoneal dialysis. Clin Kidney J 2017;10:552-9.

14. Wetmore JB, Molony JT, Liu J, et al. Readmissions following a hospitalization for cardiovascular events in dialysis patients: a retrospective cohort study. J Am Heart Assoc 2018;7:e007231.

15. Harel Z, Wald R, McArthur E, et al. Rehospitalizations and emergency department visits after hospital discharge in patients receiving maintenance hemodialysis. J Am Soc Nephrol 2015;26:3141-50.

16. Erickson KF, Winkelmayer WC, Chertow GM, et al. Physician visits and 30-day hospital readmissions in patients receiving hemodialysis. J Am Soc Nephrol 2014;25:2079-87.
17. Rosenbaum PR, Rubin DB. The central role of the propensity score in observational studies for causal effects. Biometrika 1983;70:41-55.

18. Rubin DB, Thomas N. Matching using estimated propensity scores: Relating theory to practice. Biometrics 1996;52:249-64.

19. Kendir C, van den Akker M, Vos R, et al. Cardiovascular disease patients have increased risk for comorbidity: a cross-sectional study in the Netherlands. Eur J Gen Pract 2018;24:45-50.

20. Valderas JM, Starfield B, Sibbald B, et al. Defining comorbidity: implications for understanding health and health services. Ann Fam Med 2009;7:357-63.

21. Iadecola C, Anrather J. The immunology of stroke: from mechanisms to translation. Nat Med 2011;17:796-808.

22. Javidi E, Magnus T. Autoimmunity after ischemic stroke and brain injury. Front Immunol 2019;10:686.

23. Moore KJ, Tabas I. Macrophages in the pathogenesis of atherosclerosis. Cell 2011;145:341-55.

24. Steinberg D, Witztum JL. Oxidized low-density lipoprotein and atherosclerosis. Arterioscler Thromb Vasc Biol 2010;30:2311-6.

25. Glass CK, Witztum JL. Atherosclerosis. the road ahead. Cell 2001;104:503-16.

26. Opasich C, Cazzola M, Scelsi L, et al. Blunted erythropoietin production and defective iron supply for erythropoiesis as major causes of anaemia in patients with chronic heart failure. Eur Heart J 2005;26:2232-7.

27. Androne AS, Katz SD, Lund L, et al. Hemodilution is common in patients with advanced heart failure. Circulation 2003;107:226-9.

28. Goodnough LT, Nemeth E, Ganz T. Detection, evaluation, and management of iron-restricted erythropoiesis. Blood 2010;116:4754-61.

29. Collins AJ, Li S, St Peter W, et al. Death, hospitalization, and economic associations among incident hemodialysis patients with hematocrit values of 36 to $39 \%$. J Am Soc Nephrol 2001;12:2465-73.

Cite this article as: Chen R, Yang C, Zhu M, Chu H, Wang J, Gao B, Liu L, Jiang Y, Lin Y, Wu J, Kong G, Wang F, Zhang L, Zhao M; on behalf of the China Kidney Disease Network Work Group. Association of cardiovascular disease with 30-day hospital readmission in Chinese patients receiving maintenance dialysis. Ann Transl Med 2021;9(8):617. doi: 10.21037/atm-20-2367 


\section{Supplementary}

Table S1 Codes used to identify records of patients with CVD

\begin{tabular}{|c|c|}
\hline Index condition & ICD-10-CM diagnosis codes \\
\hline $\mathrm{HF}$ & $\begin{array}{l}\text { I50.003, I50.001, I50.005, I50.000x006, I50.100, I50.100, I50.102, I50.103, I50.107, I50.000, I50.907, I50.908, } \\
\text { I50.900, I97.100x004, P29.000, I11.001, I11.000, I11.900, I13.000, I13.200, I50.900x017, I97.102, I97.803, I50.103, } \\
\text { I50.901, P29.001, I50.900x018, I50.102, J81xx02, O99.417, O99.429, O75.403, O99.423, O99.402, O99.507, } \\
\text { O29.102, O74.202, O75.402, O89.102, I50.901, I50.902, I50.903, I50.904, I50.905, I50.900x007, I50.900x008, } \\
\text { I50.900x009, I50.900x010, R57.901, J81.X00, R57.000, J96.102 }\end{array}$ \\
\hline
\end{tabular}

CVD, cardiovascular disease; CHD, coronary heart disease; HF, heart failure; ICD-10-CM, International Classification of Diseases, $10^{\text {th }}$ revision, Clinical Modification.

Table S2 Operation codes used to identify records of patients with CVD

\begin{tabular}{|c|c|c|c|}
\hline Index condition & Operation & \multicolumn{2}{|c|}{ ICD-9-CM procedure codes } \\
\hline \multirow[t]{2}{*}{$\mathrm{CHD}$} & CAG & $88.55001,88.56001,88.57002$ & $\begin{array}{l}88.5500,88.5500 \times 02,88.5600,88.5600 \times 002, \\
88.5700,88.5701,88.5700 \times 003,88.5900\end{array}$ \\
\hline & $\mathrm{PCl}$ & $36.06003,36.06004,36.07003$ & $\begin{array}{l}36.0600 .36 .0601,36.0602,36.0700,36.0700 \times 004 \text {, } \\
36.0701\end{array}$ \\
\hline
\end{tabular}

CVD, cardiovascular disease; CHD, coronary heart disease; CAG, coronary angiography; PCI, percutaneous coronary intervention; CABG, coronary artery bypass grafting; ICD-9-CM, International Classification of Diseases, $9^{\text {th }}$ revision, Clinical Modification.

Table S3 Prescription drugs categories used to identify records of patients with CVD

\begin{tabular}{ll}
\hline Index condition & Drug category \\
\hline $\mathrm{CHD}$ & Organic nitrate esters \\
$\mathrm{HF}$ & Cardiac glycosides, phosphodiesterase inhibitors, adrenergic, and dopaminergic drugs \\
Stroke & Antihypertensive agents, almitrine-raubasine combination, calcium channel blockers, antimelancholic \\
& agents, peripheral vasodilators, antivertigo medication
\end{tabular}

CVD, cardiovascular disease; CHD, coronary heart disease; HF, heart failure. 